\title{
A Comparative Analysis of Different Routing Scheme in Opportunistic Network
}

\author{
Minakshi $^{1}$ and Sona Malhotra ${ }^{2}$ \\ ${ }^{1}$ Mtech Student, UIET, Kurukshetra University, 136119, Kurukshetra, Haryana ,India \\ ${ }^{2}$ Assistant professor, UIET, Kurukshetra University, 136119, Kurukshetra, Haryana ,India
}

\begin{abstract}
Opportunistic Networks are pure wireless networks in which no direct communication is present between nodes In OPPNET no base station is used for further communication. Data transmission takes place through intermediate nodes. These intermediate nodes uses store-carry-forward model for data transmission. In opportunistic network numbers of routing protocols are presented. Routing protocols are used for data transmission in network. In this paper first introduce Opportunistic network in detail then performs a comparative analysis on different routing protocols.
\end{abstract}

Keywords: store -carry forward, opportunistic, bundle, routing etc.

\section{INTRODUCTION}

Opportunistic networks represent a class of infrastructure-less wireless framework that support the usefulness of networks encountering regular and extended durable partitions. OPPNETs are expected to manage situations including heterogeneity of guidelines, irregular network between nearby nodes. The key issue of directing for OPPNET is to locate an entrepreneurial availability between the nodes and transmit information to the nodes when they meet with each other if conceivable [2] [3]. A few techniques have been proposed to accomplish astute correspondence in such tested systems, attempting to accomplish the higher conveyance proportion with the shorter conveyance delay. Each of them has its own advantages and disadvantages.

In Opportunistic networks the network may not be connected at any given point of time. The packets are delivered in a store - carry - forward model. Every node beside the lane receives the packets starting the preceding node as it comes in contact with that particular node. After that this node stores the packet locally until it encounters next intermediate node. And when encounter occurs the packet is sent to next intermediate node. This procedure is followed until the destination is reached.

When two nodes encounter simultaneously, they may swap the packets and such an opportunity is known as encounter. In case of traditional networks, the system is associated at any given position of instance. Therefore there are no partitions in such a network. But the traditional network fails to consider several real life applications like wildlife and habitat monitoring, deep space communication, underwater infrastructure etc. That's why the delay tolerant network comes into existence, to take into account the above applications [4] [5].

1.1 Challenges

Routing in OPPNETs face many challenges due to disconnections among nodes such as:

- Contact schedules: Nodes in a OPPNET can communicate only when they are in radio range of each other. Since nodes are highly mobile, end to end path between pair of nodes may or may not exist at any given point of time. Two types of contact are scheduled and opportunistic. Scheduled contacts involve storing the information until the receiver receives it at sending rate. In opportunistic contacts, sender and receiver exchange information when they happen to be in range of each other [1].

- Storage Capacity: The messages to be forwarded have to be stored in buffer for long periods of time to take care of disconnections among nodes. Therefore intermediate nodes must have enough buffer space to hold all the 
packets that have been waiting for long for an encounter to occur [2]. Messages are forwarded to intermediate nodes along a path that leads to destination. If the OPPNET nodes are storage constrained, may result in overflow of buffer space and hence lead to packet droppings, resulting in packet loss.

- Network Capacity: Quantity of information traded between two nodes, relies on upon both the connection innovation and the length of time of the contact. Regardless of the possibility that the interval is definitely known, it may not be conceivable to anticipate the limit because of vacillations in the information rate [2]. In the event that the volume of movement increments because of expansive no. of messages being traded, the contact limit gets to be vital.

- Energy and Processing Power: Nodes consume energy in sending, receiving, storing messages and performing computations. Thus routing strategies sending fewer bytes and performing less computation are more energy efficient. OPPNET nodes having small processing capability in terms of CPU and memory cannot run complex routing protocols [2].

\subsection{Architecture}

OPPNET architecture implements store \& forward message overlaying so as to exchange another convention layer called Bundle Layer [1] on the highest point of heterogeneous locale particular lower layers with the goal that application projects can convey over various regions [4]. Bundles are also called messages. Custody transfers support node to node retransmission in bundle layer. Protocol stack for OPPNET adopted by NASA's Disruption Tolerant Networking Project is shown below:

Layered architecture of OPPNET has following sections [6]:

Link Layers: In OPPNET lower level Layers Data join layer, Internet Layer, Transport Layer are same as that of which are available in TCP/IP. The elements of these layers are same as in TCP/IP convention suite.

- $\quad$ Bundle Layer: Bundle Layer gives join between lower level connection Layers and Application layer. Information is transmitted in the system as groups and it is transmitted by Bundle Layer.

- $\quad$ Application Layer: Application Layer gives interface to the end clients. End clients can convey to different Layers through Application Layer. In application Layer different conventions are available like FTP, Telnet, and DNS and so on.

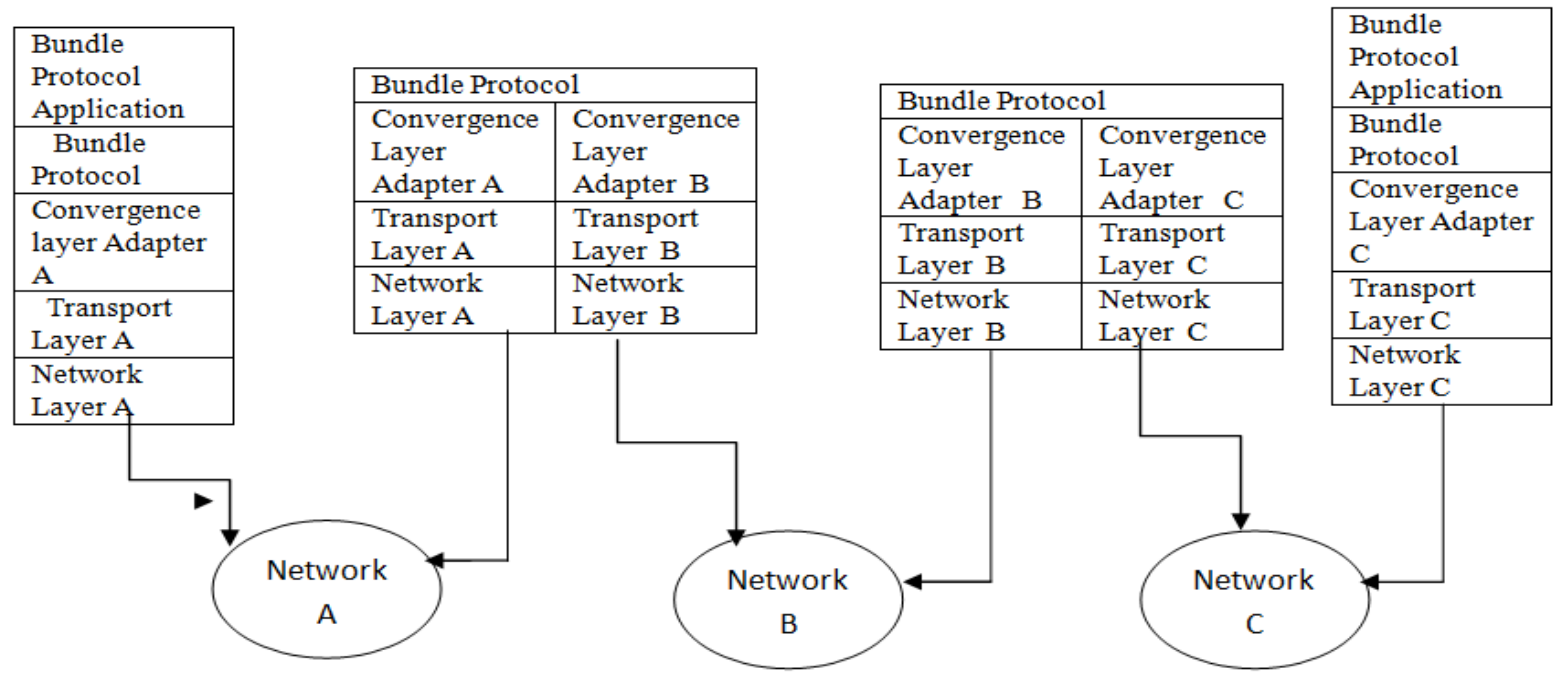

Fig. 1: OPPNET Bundle Architecture [6]

\subsection{Applications}

Some of the real life applications of Opportunistic networks comprising of wireless nodes whether mobile or stationary are listed below: 
- Mobile Adhoc Networks: Wireless sensors and mobile smart phones are used to track the locations of soldiers in military battlefield networks, who are equipped with wearable sensors on their body.

- Mule Networks: OPPNETs using sensor nodes track the position of animals in wildlife such as ZebraNet for tracking Zebras and Carrier Pigeons developed by Bergen Linux User Group for tracking pigeons by attaching sensors on their body.

- Vehicular Networks: DakNet enables the use of internet applications in rural areas with no infrastructure. It can be used when wireless directional radio is not economically feasible. DakNet is now commercialized by FirstMileSolutions in India. OpTraCom infrastruture is used in public transportation for pollution monitoring, gathers envrionmental data and operating data of the public transportation provider (e.g. status of railroad switches, diagnostic data of vehicles.

- Inter Planetary Networks: In Deep Space Networks, how information is exchanged between terrestrial objects separated by huge distances in space. The Internet Society's IPN Special Interest Group's InterPlaNetary (IPN) Internet is a OPPNET. IPN described how a message might be sent from Earth to Mars.

- Acoustic Underwater Networks: Using forestry and underwater sensors, measurements regarding temperature, intensity of natural lighting, air pressure, fire hazards, radiation levels, chemical contamination in the soil or the water can be gathered.

\subsection{Routing Strategies}

Routing schemes for opportunistic networks have been categorized as given below:

- Direct Delivery: The source node carries data with it until it directly comes in contact of destination. The message is relayed to destination only not to any intermediate node. Number of required for message delivery is only one. Direct Delivery scheme utilizes minimum resources since each packet is transmitted at most once. However it suffers from long delivery delays and shows poor performance [7] .

- Epidemic: Epidemic convention [8] ensures that a message achieves destination by spreading the message in Omni-bearings, much the same as an infection spreading a pandemic malady. In the event that a node experiences another node then them two trade messages which the other one does not have. Since the nodes have a constrained measure of support and vitality, pandemic convention devours a considerable measure of battery because of an extensive number of message. This prompts a high overhead cost.

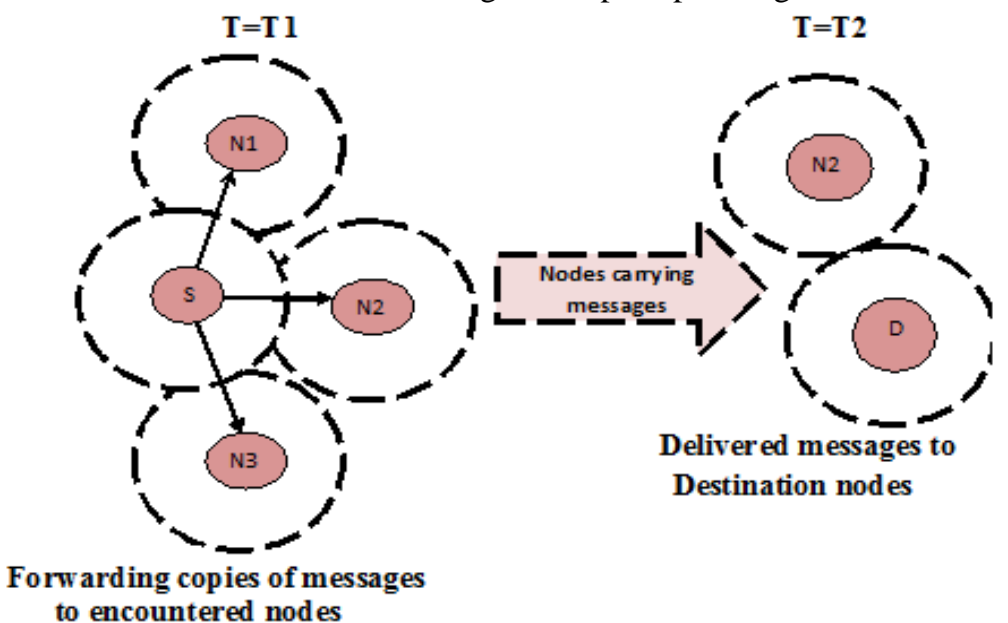

Fig.2. Epidemic Routing

- Spray and Wait: This plan [9] has two stages Spray stage and Wait stage. In splash stage, a node disperses message duplicates into the system. I hold up stage, node conveying the message duplicate stores message 
duplicate in its cradle and holds up till it gets experienced with destination node. This plan restrains the most extreme number (L) of message reproductions in the system. It has two variants source shower and hold up, Binary splash and hold up. In source shower and hold up, just source node splashes a solitary duplicate of message to first L-1 experienced nodes. The second form is Spray and Wait Binary. Every node advances a large portion of the quantity of message duplicates to next node it interacts with. All nodes conveying message are left with only a solitary duplicate of message with them. Presently these nodes hold up until they straightforwardly come in contact to the destination node, with the goal that they can exchange the planned message. The twofold form is superior to the Source, as the scattering of message is quick in the system.

- Prophet: Probabilistic Protocol utilizing History of Encounters and Transitivity [14] utilizes the authentic data of experience between nodes keeping in mind the end goal to register the conveyance consistency of each node. Every node keeps up a table for the conveyance consistency of the considerable number of nodes for every one of the destinations. At the point when any node interacts with another, then this data is exchanged. It likewise utilizes the transitive property of information to choose to the best node to forward the message. A higher conveyance proportion is watched when contrasted and scourge under meager thickness situation.

\section{RELATED WORK}

In [14] authors proposed routing principle in opportunistic network using generic algorithm based on the use of a high-dimensional Euclidean space. In this paper authors provide an analysis and the large scale evaluation of proposed routing scheme in the context of ambient networking by replaying real mobility traces. In [15] studied commons mobility models and focused on study of rate and distribution of inter connect times. In [16] presented Heterogeneous Community-based Random Way-Point (HC-RWP) mobility model that can generate synthetic traces.

In [17] examined the routing protocols for delay and disruption tolerant networks leverage epidemic-style algorithms that trade off injecting many copies of messages into the network for increased probability of message delivery. To this problem author proposed a new opportunistic network routing algorithm called Encounter-Based Routing (EBR), which maximizes delivery ratios while minimizing overhead and delay. In [18] authors gave the path routing which is optimistic and congestion control in wireless mesh network with intra session network coding. They took a different scenario where both opportunistic routing and network coding are used.

In [19] proposed an opportunistic scheduling approach to increase the throughput of next generation wireless networks for many users. Opportunistic scheduling algorithm increases the throughput by channel variation and multiple user diversity. In [20] studied the area of routing in delay-tolerant networks and presents a system for classifying the proposed routing strategies. The author presents two properties that can be used to classify delaytolerant routing strategies: replication and knowledge. In [21] presented a multi objective routing decision making model to satisfy different users and application. Authors need to use all possible information as the basis for routing decision, routing knowledge is divided into three categories: node attributes, contact attributes, message attributes

In [22] proposed an interest based mobility model and routing methods. it follows the routing strategy which is first store then carry and then forward the messages. In [23] presented the study of impact of mobility model like random walk, gauss Markova, and random waypoint on the social structure formation on OPPNET. They concluded that method of forming social structure between mobile nodes affects the density of social structure formation.

\section{COMPARISON BETWEEN DATA DELIVERY SCHEMES}

From the various data delivery schemes it has identified the various advantages and Disadvantages to each other. A comparison for this is discussed in Table I. Table I represents the advantages and disadvantages of various routing schemes. 


\section{TABLE I COMPARING OF VARIOUS ROUTING SCHEMES}

\begin{tabular}{|l|l|l|}
\hline \multicolumn{1}{|c|}{ Routing Scheme } & \multicolumn{1}{c|}{ Advantages } & \multicolumn{1}{c|}{ Disadvantages } \\
\hline $\begin{array}{l}\text { Epidemic } \\
\text { routing Scheme }\end{array}$ & $\begin{array}{l}\text { Easiest, Simple, Flooding } \\
\text { technique }\end{array}$ & $\begin{array}{l}\text { No knowledge about the network or the nodes, Inefficient } \\
\text { use of power, bandwidth \& buffer at each node }\end{array}$ \\
\hline Direct-Contact & $\begin{array}{l}\text { Simple, Do not consume } \\
\text { additional resources \& makes } \\
\text { no additional copies of data }\end{array}$ & Delivery delay can be extreme large \\
\hline One-hop Relay routing Scheme & Successful delivery of data & High cost of data delivery delay \\
\hline $\begin{array}{l}\text { Routing based on knowledge } \\
\text { Oracle Scheme }\end{array}$ & $\begin{array}{l}\text { Presence of knowledge } \\
\text { oracles, Better performance }\end{array}$ & Large storage constraints required \\
\hline
\end{tabular}

TABLE II ASSESSMENT OF VARIOUS PROPOSED ROUTING SCHEMES

\begin{tabular}{|l|l|l|l|l|}
\hline Techniques & Energy efficient & Packet drop rate & $\begin{array}{l}\text { Simulation } \\
\text { Environment }\end{array}$ & Referred work \\
\hline AntproPHET & High & Low & ONE Simulator & {$[24]$} \\
\hline SGBR & Medium & Medium & ONE Simulator & {$[25]$} \\
\hline Prediction-based Routing & Low & High & ONE Simulator & {$[26]$} \\
\hline Social Routing & Low & Low & ONE Simulator & {$[27]$} \\
\hline
\end{tabular}

In Table II assessments of different routing schemes proposed by various researchers has been analyzed. Proposed techniques were simulated on ONE simulator. Antprophet scheme is highly energy efficient with low packet drop rate.

TABLE III COMPARATIVE ANALYSIS OF EXISTING ROUTING PROTOCOLS

\begin{tabular}{|l|l|l|l|}
\hline Protocol & Created Messages & Throughput & Expenditure resources \\
\hline Direct contact & Single & Low & Less \\
\hline First contact & K Medium & Medium & Less \\
\hline Epidemic & N-1 High & High & High \\
\hline Spray and wait & $>$ K Medium & Medium & Medium \\
\hline
\end{tabular}

$\mathrm{N}=$ Nodes

$\mathrm{K}=$ optimal number of nodes to guarantee the delivery for Two-Hop.

\section{CONCLUSION}

Opportunistic networks are used in post disaster scenarios. To transfer messages and make communication between nodes in these situations OPPNET provides different routing protocols like epidemic, prophet, spray and wait, direct delivery and first contact routing. In this paper comparative analysis of various routing protocols are 
discussed before this also provides layered architecture of OPPNET and application areas where OPPNET may be applied. In future we try to propose an enhanced algorithm that increase delivery ratio and at same time reduce replication of messages during data transmission.

\section{REFERENCES}

[1] L. Pelusi, A. Passarella and M. Conti, "Opportunistic Networking: Data Forwarding in Disconnected Mobile Ad Hoc Networks", IEEE Communications Magazine, 44:131-141, November 2006.

[2] L. Lilien, Z. Kamal, V. Bhuse, and A. Gupta," Opportunistic Networks: The Concept and Research Challenges in Privacy and Security", In Proc. of 2006 International Workshop on Research Challenges in Security and Privacy for Mobile and Wireless Networks (WSPWN'06), Miami, Florida, USA, pp. 134-147, March 2006.

[3] J. Burgess, B. Gallagher, D. Jensen, and B. N. Levine, “ Maxprop: Routing For Vehicle-Based Disruption Tolerant Networks", In Proc. of the 25th IEEE International Conference on Computer Communications (INFOCOM' 06), Barcelona, Spain, pp. 1-11, April 2006.

[4] A. Vahdat and D. Becker, "Epidemic Routing For Partially Connected Ad Hoc Networks", Technical Report CS-2000-06, Dept. of Computer Science, Duke University, 2002.

[5] Burns, B., Brock, O. and Levine, "M V Routing and Capacity Building in Disruption Tolerant Networks", in the Proceedings of the 24th IEEE Annual Joint Conference on Computer and Communications Societies (INFOCOM 2005).

[6] Burgess, J., Gallagher, B., Jensen, D. and Levine, B. "MaxProp: Routing for Vehicle-based Disruption-Tolerant Networks", in the Proceedings of the 25th IEEE Annual Joint Conference on Computer and Communications Societies (INFOCOM 2006).

[7] P. Zhang, C. M. Sadler, S. A. Lyon and M. Martonosi, "Hardware Design Experiences in ZebraNet," in Proc. of the 2nd International Conference on Embedded Networked Sensor Systems, pp. 227-238, 2004.

[8] T. Small and Z. J. Haas, "The Shared Wireless Infostation Model: A New Ad Hoc Networking Paradigm (or Where there is a Whale, there is a Way)," in Proc. of MobiHOC, pp. 233-244, 2003

[9] Chung-Ming Huang, Kun-chan Lan and Chang-Zhou Tsai, "A Survey of Opportunistic Network", in 22nd IEEE International Conference on Advanced Information Networking and Applications, 2008.

[10] Muhammad Abdulla and Robert Simon, "A Simulation Study of Common Mobility Models for Opportunistic Network", in 41st IEEE Annual Simulation Symposium, 2008.

[11] L.-J. Chen, C. H. Yu, C. Tseng, H. Chu, and C. Chou, "A content-centric framework for effective data dissemination in Opportunistic Networks", IEEE Journal on selected Areas in Communications, 26(5), pp. 761-772, June 2008.

[12] Chen Zhou, Dai Wei, Zhang Sanfeng and Ji Yi , "An Interest Based Opportunistic Network Mobility Model and Routing Method", IEEE, 2012.

[13] Halikul Lenando, Mohamad Nazim Jambli, Kartinah Zen and Johari Abdullah, "Impact of Mobility Models on Social Structure Formation in Opportunistic Network", 12th IEEE International Conference on Trust, Security and Privacy in Computing and Communications 2013.

[14] Jeremie L., Timur F.,Vania C., "Evaluating Mobile Pattern Space Routing for Opportunistic Networks”, IEEE, 2004.

[15] Samuel C. Nelson, Mehedi Bakht, and Robin Kravets, “Encounter based routing in opportunistic networks” IEEE Infocom 2009

[16] Fabio Soldo, Athina Markopoulou and Alberto Lopez Toledo, "A Simple Optimization Model for Wireless Opportunistic Routing with Intra Session Network Coding", IEEE, 2010

[17] Yi Sui, "The Opportunistic Scheduling Model of Multiple Users Wireless Network", IEEE 2010.

[18] Evan Jones, Paul A and S. Ward, "Routing strategies for delay tolerant networks", IEEE 2010.

[19] Meng Chen and Haiquan Wang, “A Multi Objective Routing Decision Making Models for Opportunistic Network”, IEEE CCIS, 2011

[20] Chen Zhou, Dai Wei, Zhang Sanfeng and Ji Yi, "An Interest Based Opportunistic Network Mobility Model and Routing Method", IEEE, 2012.

[21] Halikul Lenando, Mohamad Nazim Jambli, Kartinah Zen and Johari Abdullah, "Impact of Mobility Models on Social Structure Formation in Opportunistic Network", 12th IEEE International Conference on Trust, Security and Privacy in Computing and Communications 2013.

[22] Mohamed.Ababou, R.Elkouch and M.Bellafkih, “AntProPHET: A New Routing Protocol for Delay Tolerant Networks”, IEEE, 2014.

[23] Tamer Abdelkader, Kshirasagar Naik, Amiya Nayak, Nishith Goel, and Vineet Srivastava, "SGBR: A Routing Protocol for Delay Tolerant Networks Using Social Grouping", IEEE Transactions On Parallel And Distributed Systems, 2012.

[24] Hao Yue, Huai-Lei Fu, Linke Guo, Yuguang Fang and Phone Lin, “An Efficient Prediction-based Routing Protocolin Delay Tolerant Networks", IEEE 2013, pp: 4471-4476

[25] Quoc Nguyen Viet, Pham Van Phuoc, Trinh Quoc Son and Lung Vu Duc , "Social Routing: A Novel Routing Protocol for Delay Tolerant Network based on Dynamic Connectivity", IEEE 2015, pp.1-6. 